\title{
Leiomyoma of External Auditory Canal
}

\author{
M. V. George ${ }^{1} \cdot$ Jamsheeda Puthiyapurayil $^{1}$
}

Received: 4 October 2015/ Accepted: 4 May 2016/Published online: 11 May 2016

(C) Association of Otolaryngologists of India 2016

\begin{abstract}
This article reports a case of piloleiomyoma of external auditory canal, which is the 7th case of leiomyoma of the external auditory canal being reported and the 2 nd case of leiomyoma arising from arrectores pilorum muscles, all the other five cases were angioleiomyomas, arising from blood vessels. A 52 years old male presented with a mass in the right external auditory canal and decreased hearing of 6 months duration. Tumor excision done by end aural approach. Histopathological examination report was leiomyoma. It is extremely rare for leiomyoma to occur in the external auditory canal because of the non-availability of smooth muscles in the external canal. So it should be considered as a very rare differential diagnosis for any tumor or polyp in the ear canal.
\end{abstract}

Keywords External auditory canal $\cdot$ Tumor $\cdot$ Leiomyoma

\section{Introduction}

Both benign and malignant tumors of smooth muscle origin can arise in the head and neck area [1]. Leiomyoma, the benign smooth muscle tumor commonly arise in uterus (fibroid) and gastrointestinal tract. Owing to the fact that little or no native smooth muscle exists in the head and neck region with the exception of the cervical esophagus and the circumvallate papillae and ductus lingualis of the tongue, noncutaneous smooth muscle tumors developing in

M. V. George

drgeorgemv@gmail.com

1 Department of Otorhinolaryngology, Jubilee Mission Medical College and Research Institute, Thrissur, Kerala, India this region are believed to take origin primarily from the smooth muscle forming blood vessel walls. Within head and neck, the most common sites are oesophagus and pharynx [2]. Leiomyoma may also arise from erector pili muscle found in skin and less frequently develop in deep soft tissues. Leiomyomas of external auditory canal is extremely rare. Till now, only six cases of external auditory canal leiomyomas have been reported in literature. In this report we present another case of external auditory canal leiomyoma which was successfully excised.

\section{Case Report}

A 52 years old male presented in our OPD with complaints of right ear canal mass and decreased hearing of 6 months duration. There was no history of otorrhoea, otalgia, trauma, tinnitus, headache or vertigo. On clinical examination, the right external auditory canal was obliterated by a smooth skin-lined, non-pulsatile swelling arising from the posterior part of external auditory canal without any post aural extension. On probing, the mass was non-tender and didn't bleed. Pure tone audiogram revealed a mild right sided conductive hearing loss. Contrast enhanced CT scan revealed a well defined approximately $1.8 \times 1.6 \mathrm{~cm}$ peripherally enhancing lesion at external auditory canal on right side without any intracranial or middle ear space extension (Fig. 1).

Patient underwent excision biopsy of the mass under general anesthesia. The mass was explored via an end aural approach. The soft tissue mass was seen arising from the posterior wall of bony external auditory canal. Intraoperatively minimal erosion of the posterior wall of the bony external auditory canal was noted. Desquamated epithelium seen accumulated medially in the external auditory 


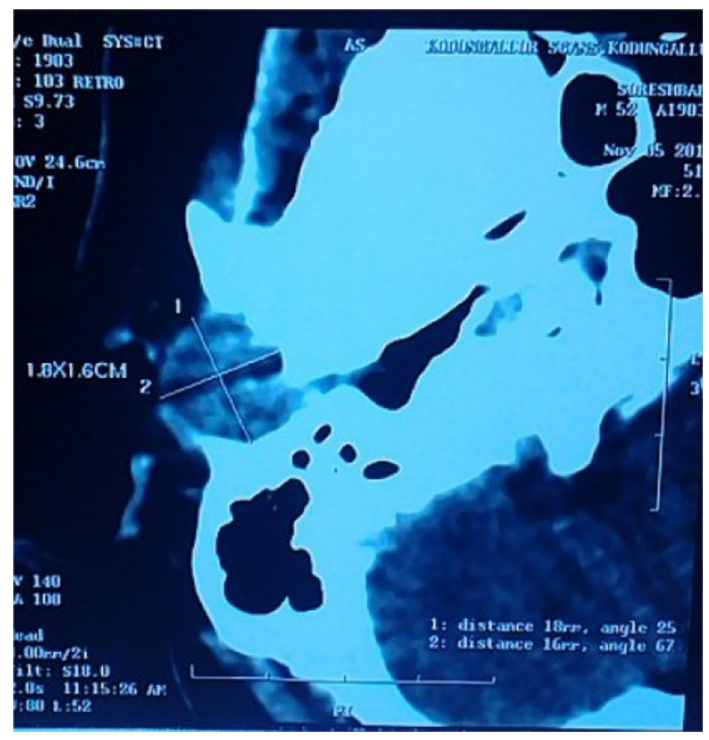

Fig. 1 CECT showing mass in EAC

canal was removed completely. Tympanic membrane was intact. The external auditory canal skin was well preserved during the procedure. Histopathological examination result was reported as leiomyoma with degenerative changes. Histologically, the neoplasm was composed of sheets and fascicles of spindle cells separated by fibro collagenous stroma with myxoid change and hyalinization. There was focal nuclear palisading but no mitosis noted. Postoperative period was uneventful. During the follow-up visit otoscopic examination revealed an intact tympanic membrane and a well healed external auditory canal. Hearing improved and there was no recurrence after 1 year followup.

\section{Discussion}

Leiomyomas may be categorized into the following 4 types:

\section{Multiple piloleiomyomas \\ Solitary piloleiomyoma \\ Angioleiomyoma (solitary) \\ Genital leiomyoma (solitary)}

This classification reflects the most logical origin of the smooth muscle tumor and corresponds to the histologic or anatomic site where the leiomyomas are found. Piloleiomyomas are believed to arise from the arrector pili muscle of the pilosebaceous unit, whereas angioleiomyomas originate from smooth muscle within the walls of arteries and veins (i.e., tunica media of blood vessels). Leiomyomas derived from the dartos muscle of the scrotum and the labia majora, as well as those derived from the erectile muscle of the nipple, are classified as genital leiomyomas [3]. Leiomyomas are usually not larger than $1-2 \mathrm{~cm}$ in greatest dimension and are composed of fascicle of spindle cell [4].

Leiomyomas are also reported in auricle [5], pre auricular region [6], nasal cavity [7], lip [8] etc. in head and neck region. A more detailed study is given by Wang et al. [9].

Leiomyomas are most commonly found in areas of body that contain abundant smooth muscle, usually gastrointestinal tract and female genital tracts. Smooth muscle tumors of head and neck region is a rare lesion [2]. Low incidence is due to scarcity of smooth muscle in this area, which is limited to vessel walls, erector pili muscle of hair follicle, oesophagus, and posterior wall of trachea (Fig. 2).

In literature, only six cases of leiomyoma of external auditory canal have been reported. The first report was by Petschenik et al. [10] published in January 1996. That patient's entire external auditory meatus was occluded, causing a maximal conductive hearing loss on the affected side. That did not appear to be a typical aural polyp and carcinoma was suspected. Biopsy revealed the mass to be a leiomyoma of the external auditory canal. It was a benign tumor and was treated by complete excision.

The 2nd case was reported by Iguchi et al. [11], 1 year later. In their case, the tumor was seen arising from posterior wall of bony canal.

The 3rd case was reported by Yeh and Chao [12] from Taiwan. The patient was a 47-year-old male with left external auditory canal leiomyoma presented with conductive hearing impairment and post-auricular swelling. Tumor excision was successful via a retroauricular approach. The pathological examination demonstrated a vascular leiomyoma without malignant change.

Fourth report was by Pasqualina et al. [13]. They says that angioleiomyoma accounted for $5 \%$ of all soft benign

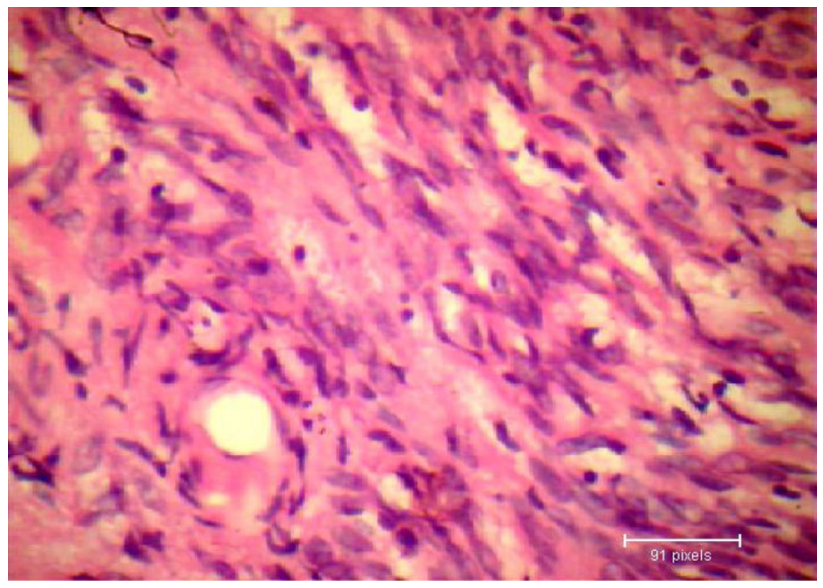

Fig. 2 Smooth muscle cells in H\&E staining (high power view) 
tissue tumors and arises from smooth muscle cells of tunica media of vascular structures. The peak incidence is between 3rd and 6th decades of life with a female preponderance.

Fifth report was by Wootten et al. [14]. They reported a case of leiomyoma of the EAC arising in a pediatric patient, and this was the first report in a paediatric age group.

The latest report was by Ikeda et al. [15]. They presented the report of a 37-year-old man with right auricular fullness. Preoperative findings suggested benign tumor or cholesteatoma in the right external auditory canal. They performed total resection using an endoauricular approach with transcanal endoscopic ear surgery. Histopathological and immunohistochemistry examination confirmed the diagnosis of leiomyoma of external auditory canal. They could get only reports of four cases of leiomyoma of external auditory canal. The other four cases demonstrated vascular leiomyomas. This was the first report of leiomyoma of the EAC arising from arrectores pilorum muscles.

Accumulated experience about the management of leiomyoma depends on case reports and literature reviews. The proper advocated treatment is complete excision. In the first reported case the tumor was excised, with tympanoplasty and cortical mastoidectomy was done while in the second case the neoplasm was removed via an endaural excision and in the third one, a retroauricular approach and excision was done. In our case, the tumor was excised via an endaural approach. Approach method and surgical extent depends on the site of tumor. Precise pathological examination is mandatory to exclude any malignant nature of the tumor.

\section{Conclusion}

We report a rare case of leiomyoma of external auditory canal which should be considered as a differential diagnosis for external auditory canal tumors. Although drawing conclusion about the treatment and outcome of leiomyoma is difficult, however a generally accepted treatment in these cases, is a surgical exploration and complete excision followed by histopathological confirmation and follow-up for a reasonable period.

\section{Compliance with Ethical Standards}

Conflict of interest There are no conflicts of interest.
Animal Rights This article does not contain any studies with animals performed by any of the authors.

Ethical Standard The procedure was performed in accordance with the ethical standards of the institution and being a rare case it is only reported.

Informed Consent Case report-patient's consent is taken for surgery as well as publication.

Financial Support This research received no specific grant from any funding agency, commercial or not-for-profit sectors.

\section{References}

1. Shah J, Patel S, Singh B (2012) Head and neck surgery and oncology, 4th edn. Elsevier, Philadelphia

2. Picciotti PM, Cantore I, La Greca C, Di Nardo W, Scarano E (2007) Angioleiomyoma of the external auditory canal. Am J Otolaryngol 28(4):235-237

3. Horner KL Leiomyoma-pathophysiology. In: James WD (ed). http://emedicine.medscape.com/article/1057733-overview\#a6

4. Yeh SY, Chao TK (2005) Leiomyoma of the external auditory canal-a case report and brief review of the literature. Eur Arch Otorhinolaryngol 262(5):397-399

5. Moon H, Chin SS, Park MK (2012) Preauricular Angioleiomyoma. Korean J Audiol 16(3):138-140

6. Inoue F, Matsumoto K (1983) Vascular leiomyoma of the auricle. Arch Dermatol 119(5):445-446. doi:10.1001/archderm.1983.016 50290085024

7. Singh R, Hazarika P, Balakrishnan R, Gangwar N, Pujary P (2008) Leiomyoma of the nasal septum. Indian J Cancer 45:173-175

8. Gueiros L-A, Romañach M-J, Pires-Soubhia A-M, Pires F-R, Paesde-Almeida O, Vargas P-A (2011) Angioleiomyoma affecting the lips: report of 3 cases and review of the literature. Med Oral Patol Oral Cir Bucal 16(4):e482-e487. doi: 10.4317/medoral.16.e482

9. Wang CP, Chang YL, Sheen TS (2004) Vascular leiomyoma of the head and neck. Laryngoscope 114(4):661-665. doi: 10.1097/00005537-200404000-00012

10. Petschenik AJ, Linstrom CJ, McCormick SA (1996) Leiomyoma of the external auditory canal. Am J Otol 17:133-136

11. Iguchi H, Nakai Y, Yamane H, Goto K, Wakasa K (1997) Smooth muscle tumor of the external auditory canal. Otolaryngol Head Neck Surg 116:231-233

12. Yeh S-Y, Chao T-K (2005) Leiomyoma of external auditory canal-a case report and brief review of literature. Eur Arch Otorhinolaryngol 262:397-399

13. Pasqualina PM, Cantore I, La Greca C, Di Nardo W, Scarano E (2007) Angioleiomyoma of the external auditory canal. Am J Otolaryngol 28:235-237

14. Wootten CT, Fasig J, Kaylie DM (2007) External auditory canal leiomyoma: a pediatric case report. Int J Pediatr Otorhinolaryngol Extra 2(1):51-53. doi:10.1016/j.pedex.2006.12.006

15. Ikeda R, Tateda M, Okoshi A, Morita S, Suzuki H, Hashimoto S (2015) Transcanal endoscopic resection for leiomyoma of the external auditory canal and a review of the literature. Auris Nasus Larynx. doi: 10.1016/j.anl.2015.05.003 